\title{
Separation of type and grade in cervical tumours using non-mono-exponential models of diffusion-weighted MRI
}

\author{
Jessica M. Winfield ${ }^{1,2}$ • Matthew R. Orton ${ }^{2}$ - David J. Collins ${ }^{1,2}$ • Thomas E. J. Ind ${ }^{3}$ • \\ Ayoma Attygalle $^{4}$ - Steve Hazell ${ }^{4}$. Veronica A. Morgan ${ }^{1,2}$ - Nandita M. deSouza ${ }^{1,2}$
}

Received: 19 January 2016 /Revised: 15 April 2016/Accepted: 13 May 2016/Published online: 24 May 2016

(C) The Author(s) 2016. This article is published with open access at Springerlink.com

\begin{abstract}
Objectives Assessment of empirical diffusion-weighted MRI (DW-MRI) models in cervical tumours to investigate whether fitted parameters distinguish between types and grades of tumours.

Methods Forty-two patients (24 squamous cell carcinomas, 14 well/moderately differentiated, 10 poorly differentiated; 15 adenocarcinomas, $13 \mathrm{well} /$ moderately differentiated, two poorly differentiated; three rare types) were imaged at $3 \mathrm{~T}$ using nine b-values ( 0 to $800 \mathrm{~s} \mathrm{~mm}^{-2}$ ). Mono-exponential, stretched exponential, kurtosis, statistical, and bi-exponential models were fitted. Model preference was assessed using Bayesian Information Criterion analysis. Differences in fitted parameters between tumour types/grades and correlation between fitted parameters were assessed using two-way analysis of variance and Pearson's linear correlation coefficient, respectively. Results Non-mono-exponential models were preferred by $83 \%$ of tumours with bi-exponential and stretched exponential models preferred by the largest numbers of tumours. Apparent diffusion coefficient (ADC) and diffusion coefficients from non-mono-exponential models were significantly
\end{abstract}

Jessica M. Winfield

jessica.winfield@icr.ac.uk

1 MRI Unit, The Royal Marsden NHS Foundation Trust, Downs Road, Sutton, Surrey SM2 5PT, UK

2 Cancer Research UK Cancer Imaging Centre, Division of Radiotherapy and Imaging, The Institute of Cancer Research, 123 Old Brompton Road, London SW7 3RP, UK

3 Gynaecology Unit, The Royal Marsden NHS Foundation Trust, Fulham Road, London SW3 6JJ, UK

4 Department of Histopathology, The Royal Marsden NHS Foundation Trust, Fulham Road, London SW3 6JJ, UK lower in poorly differentiated tumours than well/moderately differentiated tumours. $\alpha$ (stretched exponential), K (kurtosis), $f$ and $\mathrm{D} *$ (bi-exponential) were significantly different between tumour types. Strong correlation was observed between ADC and diffusion coefficients from other models.

Conclusions Non-mono-exponential models were preferred to the mono-exponential model in DW-MRI data from cervical tumours. Parameters of non-mono-exponential models showed significant differences between types and grades of tumours.

Key Points

- Non-mono-exponential DW-MRI models are preferred in the majority of cervical tumours.

- Poorly differentiated cervical tumours exhibit lower diffusion coefficients than well/moderately differentiated tumours.

- Non-mono-exponential model parameters $\alpha, K, f$, and $D^{*}$ differ between tumour types.

- Micro-structural features are likely to affect parameters in non-mono-exponential models differently.

Keywords Diffusion-weighted magnetic resonance imaging · Analysis, regression - Apparent diffusion coefficient . Intravoxel incoherent motion (IVIM) $\cdot$ Cervical cancer

\begin{tabular}{ll}
\multicolumn{2}{l}{ Abbreviations } \\
DW-MRI & Diffusion-weighted magnetic resonance imaging \\
ADC & Apparent diffusion coefficient \\
EPI & Echo-planar imaging \\
FOV & Field-of-view \\
PE & Phase encoding \\
RL & Right-left \\
TE & Echo time \\
TR & Repetition time \\
SPIR & Spectral inversion recovery
\end{tabular}




$\begin{array}{ll}\text { NSA } & \text { Number of signal averages } \\ \text { ROI } & \text { Region of interest } \\ \text { DDC } & \text { Distributed diffusion coefficient } \\ \text { IVIM } & \text { Intravoxel incoherent motion } \\ \text { BIC } & \text { Bayesian information criterion } \\ \text { ANOVA } & \text { Analysis of variance } \\ \text { CV } & \text { Coefficient of variation } \\ \text { SNR } & \text { Signal-to-noise ratio }\end{array}$

\section{Introduction}

It is increasingly recognised that the observed diffusionweighted magnetic resonance imaging (DW-MRI) signal attenuation in biological tissues is not completely described by a Gaussian process [1-5]. The use of nonmono-exponential models provides a better description of the DW-MRI signal, and parameters derived from these models allow more detailed investigation of differences between tumour sub-types or inter-tumour heterogeneity [6-11] and may also provide an earlier indication of response to treatment [12, 13]. However, use of a model with a large number of additional parameters risks over-fitting the data and may be sensitive to noise characteristics of the system rather than structural properties of the tumour or normal tissue. The potential for exploiting these parameters to describe tumour phenotypes remains substantial, but data relating them to the micro-structural properties of tumours or normal tissues is limited, particularly in body applications. Owing to the complexity of tissue and tumour micro-structure within a voxel [14], these models can at least be viewed as phenomenological descriptions of the data and in addition have demonstrable value as empirical markers of tissue status [15]. Nevertheless, their relationship to tissue micro-structural properties is an important topic of ongoing exploration [16, 17].

In cervical cancer the lower apparent diffusion coefficient (ADC) in tumours compared with non-tumour epithelium provides excellent tumour-to-normal-tissue contrast and DW-MRI is routinely used in conjunction with $\mathrm{T}_{2}$-weighted imaging for tumour detection [18-20]. Previous studies have shown lower ADCs in poorly differentiated tumours than in well/moderately differentiated tumours, but attempts to distinguish between squamous cell carcinoma and adenocarcinoma using ADC estimates have yielded mixed results $[21,22]$. Use of a biexponential model has indicated a lower perfusion fraction $(f)$ and diffusion coefficient (D) in cervical tumours than in normal cervix [23]. Assessment of the relationships between fitted parameters of DW-MRI models and tumour histopathology would establish the ability of a model to detect differences between grades and types of tumour, and the potential to detect treatment effects that may not be described by the ADC derived from the mono-exponential model. The aims of this exploratory study, therefore, were to assess the performance of mono-exponential and non-mono-exponential models of the DW-MRI signal in cervical tumours and assess whether fitted parameters from these models can be used to distinguish between types and grades of tumours.

\section{Materials and methods}

Patients Forty-two consecutive patients with histologically proven cervical tumours and tumour volume at least $50 \mathrm{~mm}^{3}$ visible on DW-MRI, recruited over two years (from May 2013 to May 2015), were included in this prospective single-centre study. The study was approved by a national research ethics committee. All patients gave their written consent to participate in this study. Tumours included 24 squamous cell carcinomas (14 well or moderately differentiated, 10 poorly differentiated), 15 adenocarcinomas (13 well or moderately differentiated, two poorly differentiated), one clear cell carcinoma, one poorly differentiated carcinoma with focal squamous differentiation and for the large part neuroendocrine differentiation, and one high grade carcinoma that could not be subclassified. The latter three tumours were included in the assessment of models, but excluded from the analysis of grades and types.

Imaging protocol Each patient underwent one MRI examination. Hyoscine butylbromide $(20 \mathrm{mg})$ i.m. was administered before scanning to reduce image artefacts due to peristalsis. Patients were scanned on a Philips Achieva 3 T MR scanner using an endovaginal coil, as described previously, engineered for imaging at $3 \mathrm{~T}$ [24]. Following $\mathrm{T}_{2}$-weighted and DW images acquired transversely, coronally, and sagitally through the cervix, a sequence with nine b-values between $0 \mathrm{~s} \mathrm{~mm}^{-2}$ and $800 \mathrm{~s} \mathrm{~mm}^{-2}$ was acquired coronally through the cervix for assessment of DW-MRI models. The protocol for this sequence was as follows: single-shot EPI; $\mathrm{FOV}=100 \mathrm{~mm} \times 100 \mathrm{~mm}$; PE direction = RL; acquired matrix $($ read $)=80$; reconstructed matrix $($ read $)=224$; acquired pixel size $=1.25 \mathrm{~mm} \times 1.25 \mathrm{~mm}$; slice thickness $=2 \mathrm{~mm}$; slice gap $=0.1 \mathrm{~mm} ; 24$ slices; $\mathrm{TE}=52 \mathrm{~ms} ; \mathrm{TR}=6500 \mathrm{~ms}$; $\mathrm{b}=0,20,40,60,80,100,300,500,800 \mathrm{~s} \mathrm{~mm}^{-2} ; \Delta /$ $\delta=25.5 / 7.5 \mathrm{~ms}$; SPIR fat suppression; NSA $=1$ for $\mathrm{b}$ values $<500 \mathrm{~s} \mathrm{~mm}^{-2}$; NSA $=2$ for $b$-values $\geq 500 \mathrm{~s} \mathrm{~mm}^{-2}$; total acquisition time $=7 \min 9 \mathrm{~s}$.

Analysis Regions of interest (ROIs) were drawn on computed $\mathrm{b}=800 \mathrm{~s} \mathrm{~mm}^{-2} \mathrm{DW}$ images [25] using in-house software, with reference to the $\mathrm{T}_{2}$-weighted images. ROIs were drawn on all 
slices on which the tumour appeared. The median tumour volume was $2.3 \mathrm{~cm}^{3}$ (range $0.07 \mathrm{~cm}^{3}$ to $40.8 \mathrm{~cm}^{3}$ ). Monoexponential (Eq. 1), stretched exponential (Eq. 2), kurtosis (Eq. 3), statistical (Eq. 4), and bi-exponential (Eq. 5) models were fitted to the data at each pixel using all nine $b$-values using least-squares fits (Matlab 2014a, MathWorks Inc., Natick, MA, USA).

These five models, recently applied in DW-MRI in body applications, were investigated. The mono-exponential model (Eq. 1), is commonly applied in DW-MRI to estimate the ADC.

$$
S=S_{0} \exp (-b \mathrm{ADC})
$$

The stretched exponential model (Eq. 2) uses a distributed diffusion coefficient (DDC) and "stretching parameter" $(\alpha)$ to describe non-mono-exponential decay curves [2]. When fitting the stretched exponential model, the "stretching parameter", $\alpha$, was constrained to lie between 0 and 1 .

$S=S_{0} \exp \left(-(b \mathrm{DDC})^{\alpha}\right)$

The kurtosis model (Eq. 3) uses the first two terms of a cumulate expansion to describe non-mono-exponential decay of a DW-MRI signal [4].

$S=S_{0} \exp \left(-b \mathrm{D}_{\mathrm{K}}+\frac{1}{6} b^{2} \mathrm{D}_{\mathrm{K}}^{2} \mathrm{~K}\right)$

The statistical model (Eq. 4) describes the distribution of diffusion coefficients within a voxel using a truncated Gaussian distribution with mode $\mathrm{D}_{\mathrm{s}}$ and scale parameter $\sigma$, where $\Phi$ is the error function [3]. In this study, the fitted parameters $D_{s}$ and $\sigma$ were also reformulated to obtain the mean $\left(D_{s^{\prime}}\right)$ and standard deviation $\left(\sigma^{\prime}\right)$ of the distribution of diffusion coefficients within a voxel using results derived elsewhere for a truncated normal distribution [26].

$S=S_{0}\left(\frac{1+\Phi\left(\frac{\mathrm{D}_{\mathrm{s}}}{\sigma \sqrt{2}}-\frac{b \sigma}{\sqrt{2}}\right)}{1+\Phi\left(\frac{\mathrm{D}_{\mathrm{s}}}{\sigma \sqrt{2}}\right)}\right) \exp \left(-b \mathrm{D}_{\mathrm{s}}+\frac{1}{2} b^{2} \sigma^{2}\right)$

The bi-exponential model (Eq. 5), also called intravoxel incoherent motion (IVIM), describes a fast component of the DW-MRI signal (D*), associated with perfusion, and a slow component (D), associated with diffusion [1]. The term $f$ in Eq. 5 describes the perfusion fraction. When fitting the bi-exponential model, starting values of $\mathrm{D}, f$, and $D^{*}$ were determined from a least-squares fit of a mono-exponential curve to the signal at the highest three b-values and another mono-exponential curve fitted to the remaining signal at the lower b-values; these starting values were used for the least-squares fit of the biexponential curve to the data at all nine b-values.

$S=S_{0}\left(f \exp \left(-b \mathrm{D}^{*}\right)+(1-f) \exp (-b \mathrm{D})\right)$

The diffusion coefficients from the non-mono-exponential models (DDC, $\mathrm{D}_{\mathrm{K}}, \mathrm{D}_{\mathrm{s}}, \mathrm{D}_{\mathrm{s}^{\prime}}$, and $\mathrm{D}$ ) may provide comparable information to ADC. For all the above models the signal is expected to decrease with increasing b-value; therefore, any pixels where the signal increased between any pair of successive b-values were excluded from the analysis. Pixels with a fitted $\mathrm{S}_{0}$ below a threshold value in monoexponential fits were also excluded; a threshold of 20 was chosen as this was the mean pixel value in background regions at the edges of the images far from the coil. The median number of pixels included in the analysis per tumour was 4411 (range 56 to 56,722). The median value of each fitted parameter, calculated for each tumour, was used for subsequent analysis in order to reduce sensitivity to outlier values. Statistical preference for the five models was quantified using the Bayesian information criterion (BIC), which penalises additional parameters in the models, as described in Eq. 6 where $L(\theta)$ is the value of the maximised likelihood objective function for a model with $k$ parameters fit to $N$ data points [27].

$\mathrm{BIC}=-2 \log L(\theta)+k \log (N)$

The preferred model, defined as the model with the lowest (i.e. most negative) BIC, was determined for each pixel. The preferred model for each tumour was defined as the model preferred by the largest number of pixels in the tumour. Two-way analysis of variance (ANOVA, Matlab 2014a) was used to assess differences between types and grades of tumours for each of the fitted parameters. No correction for multiple comparisons was applied owing to the expected correlation between many of the fitted parameters. Pearson's linear correlation coefficient (Matlab 2014a) was used to assess correlation between ADC and diffusion coefficients from non-mono-exponential models and to assess correlation between parameters within each non-mono-exponential model.

Tumour grade and type were determined from histopathological analysis of post-surgery samples $(n=12)$ or preimaging cone biopsy data $(\mathrm{n}=30)$, which samples a substantial $\left(\sim 1 \mathrm{~cm}^{3}\right)$ tumour volume.

\section{Results}

The well-differentiated squamous cell carcinoma visualised in Fig. 1 demonstrates restricted diffusion (bright signal on the diffusion-weighted image and corresponding dark region on 

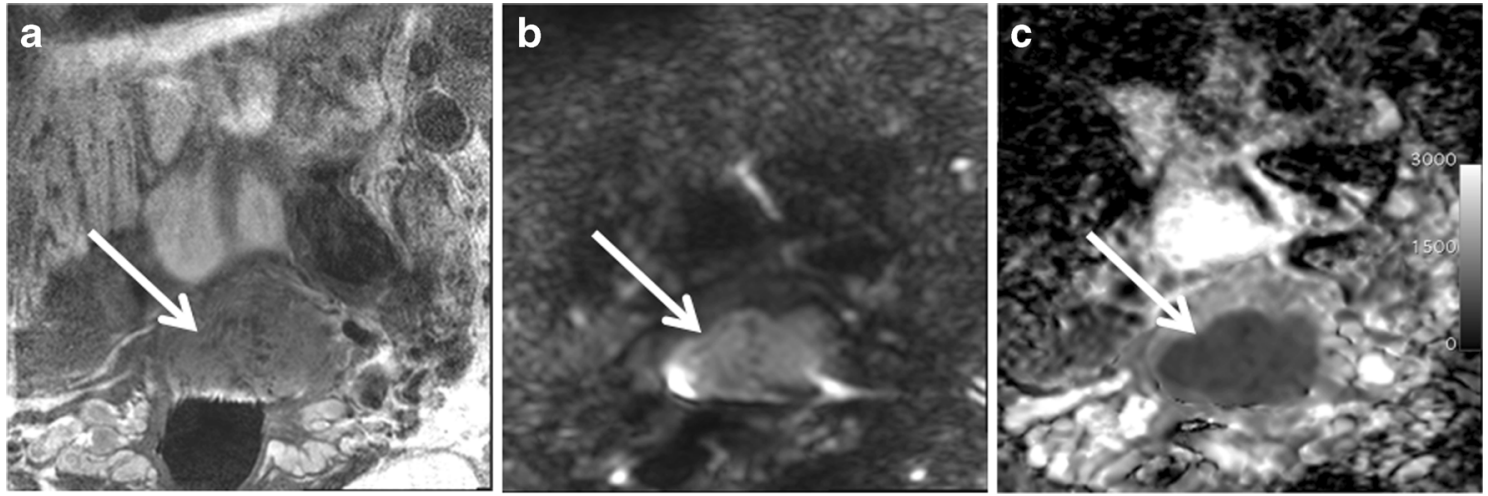

d

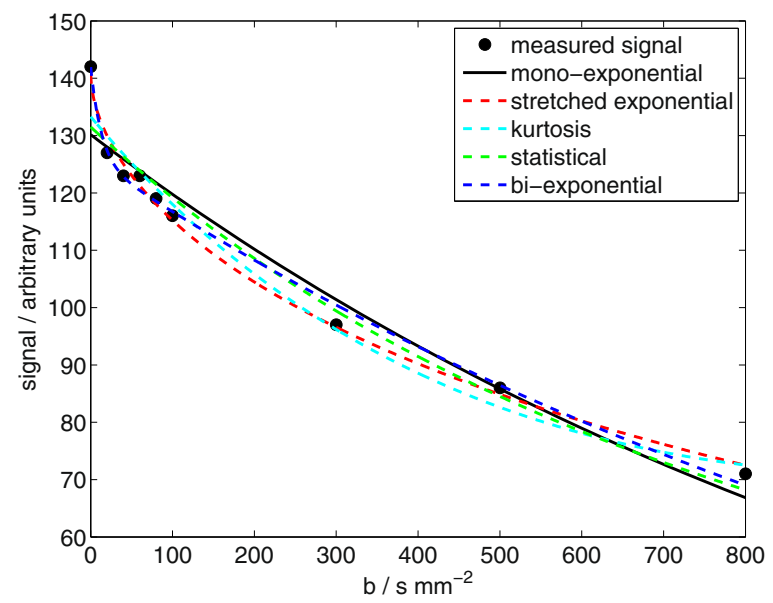

Fig. 1 a $\mathrm{T}_{2}$-weighted image, $\mathbf{b}$ diffusion-weighted image $\left(b=800 \mathrm{~s} \mathrm{~mm}^{-2}\right), \mathbf{c}$ ADC map from a well-differentiated squamous cell carcinoma. $\mathbf{d}$ Measured signal and fitted curves from one pixel near the centre of the tumour shown in (a-c)

the ADC). Example curve fits from one pixel (Fig. 1d) show clear non-mono-exponential signal attenuation with increasing b-value, which is better described by the non-monoexponential models (dashed lines in Fig. 1d) than the monoexponential model (solid black line in Fig. 1d).

The percentages of tumours where BIC analysis indicated that the bi-exponential and stretched exponential models were preferred by the majority of pixels were $43 \%$ and $36 \%$, respectively (Table 1 ). Overall, nonmono-exponential models were preferred by $83 \%$ of tumours, with the mono-exponential model preferred by only $17 \%$. When looking at the number of tumours of each type and grade where each model was preferred, there is no clear preference of a particular type or grade for any of the models. When considering the tumours where each of the models was preferred, the percentage of pixels preferring that model ranged from $26 \%$ to $48 \%$. This indicates a moderate preference for the dominant model, but also demonstrates that there are many pixels within each tumour that prefer models other than the dominant model.
Results from ANOVA, summarised in Table 2, showed that $\alpha$ from the stretched exponential model, $\mathrm{K}$ from the kurtosis model, and $f$ and $\mathrm{D}^{*}$ from the bi-exponential model were significantly different between types of tumour (squamous cell carcinoma versus adenocarcinoma) (Fig. 2). ADC from the mono-exponential model, DDC from the stretched exponential model, $\mathrm{D}_{\mathrm{K}}$ from the kurtosis model, $\mathrm{D}_{\mathrm{s}^{\prime}}$ from the statistical model and $\mathrm{D}$ from the bi-exponential model were significantly different between tumour grades (well/moderately differentiated versus poorly differentiated) (Fig. 3).

Assessment of correlation between ADC and other diffusion coefficients showed that DDC, $\mathrm{D}_{\mathrm{K}}, \mathrm{D}_{\mathrm{s}^{\prime}}$ (mean), and D were strongly correlated with ADC (Fig. 4, $\mathrm{r}>0.9, p<10^{-6}$ in all cases). Correlation between $\mathrm{D}_{\mathrm{s}}$ (mode) and ADC was less strong $(\mathrm{r}=0.58, p=0.0001)$. When assessing correlation of parameters within each model, no correlation was observed between $\alpha$ and DDC from the stretched exponential model, $\sigma$ and $D_{s}$ from the statistical model, nor between either $f$ and $\mathrm{D}$ or $\mathrm{D}^{*}$ and $\mathrm{D}$ from the bi-exponential model (Table 3 ). 
Weak correlation was observed between $f$ and $\mathrm{D}^{*}$ and between $f \mathrm{D}^{*}$ and $\mathrm{D}$ from the bi-exponential model. Negative correlation was observed, however, between $\mathrm{K}$ and $\mathrm{D}_{\mathrm{K}}$ from the kurtosis model, and strong positive correlation was observed between $\sigma^{\prime}$ and $\mathrm{D}_{\mathrm{s}^{\prime}}$ from the reformulated statistical model.

\section{Discussion}

The preference for non-mono-exponential models in the majority of tumours indicates that these models provide a better description of the DW-MRI data in cervical tumours than a mono-exponential model. The absence of a clear preference for a particular model within each grade and type of tumour indicates that the models assessed here are applicable across all tumours and the choice of model is not driven purely by characteristics of a particular tumour grade or type. The preference for a non-mono-exponential model fit for the data from these tumours is consistent with results from other studies: a study in squamous cell carcinoma of the head and neck demonstrated that the kurtosis model provided a better fit to DW-MRI data than the mono-exponential model in primary tumours and in metastatic lymph nodes [7] and a study in advanced ovarian cancer demonstrated a preference for the stretched exponential model over the mono-exponential model in the majority of primary and metastatic lesions in DW-MRI data both pre- and posttreatment [11]. Clinical studies comparing various models for DW-MRI data from prostate cancer have also demonstrated a better fit in malignant as well as benign regions using the bi-exponential model [6] or the kurtosis model [17] compared with the mono-exponential model. In contrast, a study in normal and malignant breast tissue found that DW-MRI data from approximately half of all voxels preferred a mono-exponential model while either a stretched exponential or bi-exponential model was preferred in just under half of the voxels analysed [28]. It is, however, important to note that the mono-exponential model can be considered a special case of the other models applied here (using e.g. $\alpha=1, \mathrm{~K}=0, f=0$ ), as has been pointed out in previous studies [13], suggesting that tumours that prefer the mono-exponential model are not disadvantaged by the use of other models, although the additional parameters would be redundant if the mono-exponential model were preferred in all tumours. The stretched exponential, kurtosis, and statistical models describe the departure of the DW-MRI signal decay from mono-exponential behaviour, which may be due to intra-voxel heterogeneity or non-Gaussian diffusion processes, either of which may be related to the observed separation of types and grades of tumours. 
Table 2 Assessment of differences in each fitted parameter between types and grades of tumour using two-way ANOVA. (s.d. standard deviation) $* p<0.05$

\begin{tabular}{llll}
\hline Model & Parameter & $p$-value from ANOVA (type) & $p$-value from ANOVA (grade) \\
\hline Mono-exponential & ADC & 0.1 & $\mathbf{0 . 0 2} *$ \\
Stretched exponential & DDC & 0.1 & $\mathbf{0 . 0 2} *$ \\
& $\alpha$ & $\mathbf{0 . 0 1} *$ & 0.7 \\
Kurtosis & $\mathrm{D}_{\mathrm{K}}$ & 0.2 & $\mathbf{0 . 0 2} *$ \\
& $\mathrm{~K}$ & $\mathbf{0 . 0 3} *$ & 0.07 \\
Statistical & $\mathrm{D}_{\mathrm{s}}$ (mode) & 0.2 & 0.1 \\
& $\sigma$ (scale parameter) & 0.7 & 0.2 \\
& $\mathrm{D}_{\mathrm{s}^{\prime}}$ (mean) & 0.2 & $\mathbf{0 . 0 2} *$ \\
& $\sigma^{\prime}$ (s.d.) & 0.5 & 0.07 \\
Bi-exponential & $\mathrm{D}$ & 0.9 & $\mathbf{0 . 0 1} *$ \\
& $f$ & $\mathbf{0 . 0 0 0 3} *$ & 0.9 \\
& $\mathrm{D}^{*}$ & $\mathbf{0 . 0 0 2} *$ & 0.4 \\
& $f \mathrm{D}^{*}$ & 0.3 & 0.05 \\
\hline
\end{tabular}

The differences in fitted parameters between types or grades of tumours observed in this study indicate that these parameters are sensitive to micro-structural properties of the tumours. In this study, tumour grade was reflected by the diffusion coefficients; this was the case whether considering simply ADC or the diffusion coefficients from non-mono-exponential models (DDC, $\mathrm{D}_{\mathrm{K}}, \mathrm{D}_{\mathrm{s}}$, and D). ADC could, therefore, be considered sufficient to describe tumour grade, but other parameters from the nonmono-exponential models $\left(\alpha, \mathrm{K}, f, \mathrm{D}^{*}\right)$ provided further information that differentiated between tumour types. The use of non-mono-exponential models thus provides a more complete characterisation of the tumours than provided by ADC alone. The lower ADC observed in poorly differentiated tumours compared with well/moderately differentiated tumours is in agreement with results of
Fig. 2 Differences between types of tumour (squamous cell carcinoma $(n=24)$ versus adenocarcinoma $(\mathrm{n}=15))$ in (a) $\alpha$ from the stretched exponential model, $\mathbf{b} \mathrm{K}$ from the kurtosis model, and $\mathbf{c} f$ and $\mathbf{d} \mathrm{D}^{*}$ from the bi-exponential model a

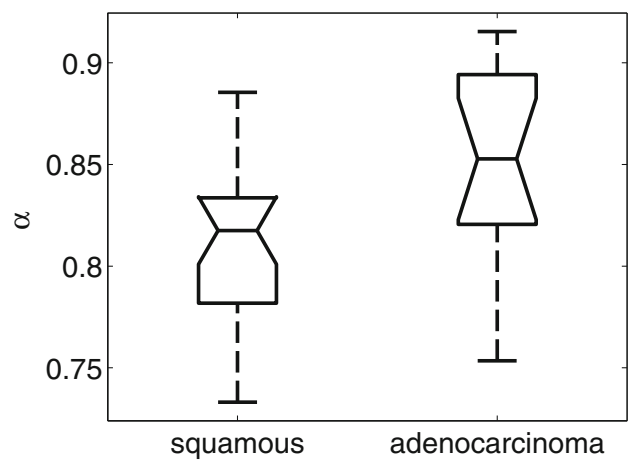

C

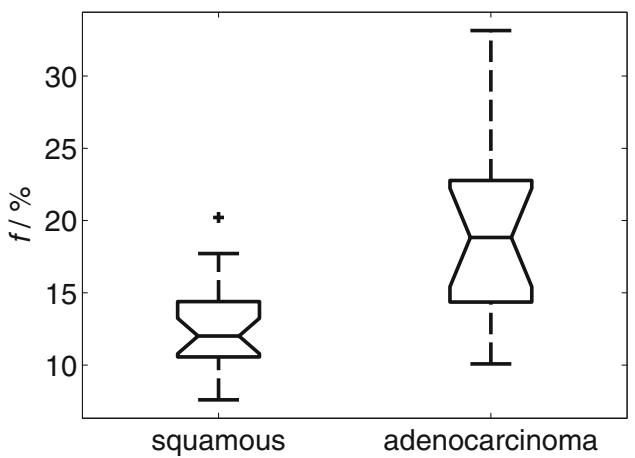

b

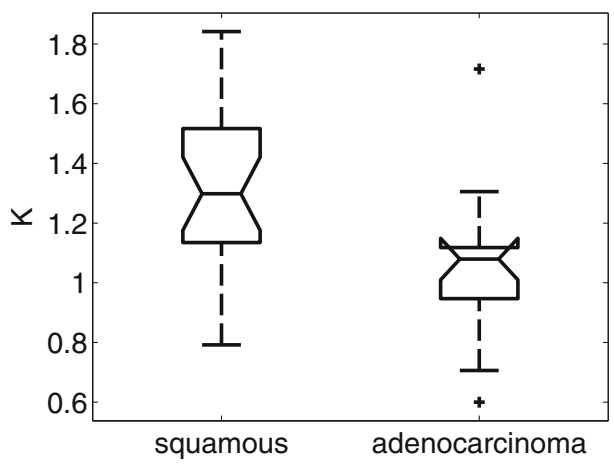

d

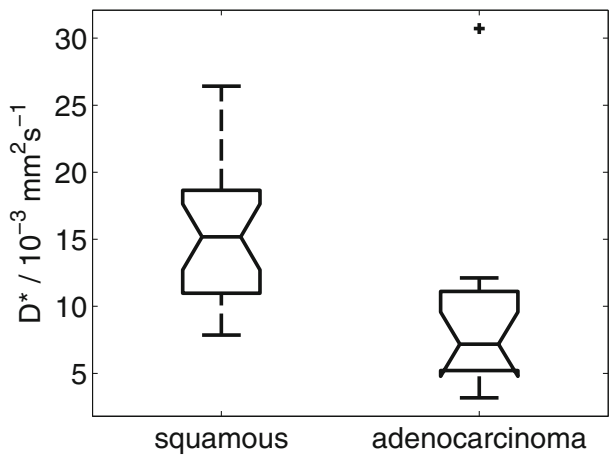


Fig. 3 Differences between grades of tumour (well/moderately differentiated $(\mathrm{n}=27)$ versus poorly differentiated $(\mathrm{n}=12))$ in a ADC from the mono-exponential model, $\mathbf{b}$ DDC from the stretched exponential model, $\mathbf{c} \mathrm{D}_{\mathrm{K}}$ from the kurtosis model, $\mathbf{d} \mathrm{D}_{\mathrm{s}^{\prime}}$ from the statistical model, and $\mathbf{e} \mathrm{D}$ from the bi-exponential model a

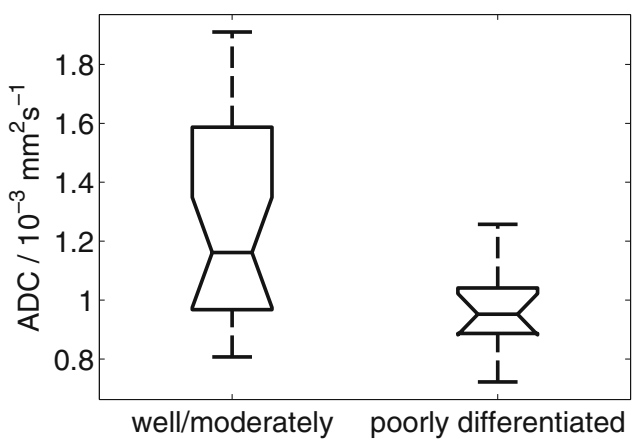

C

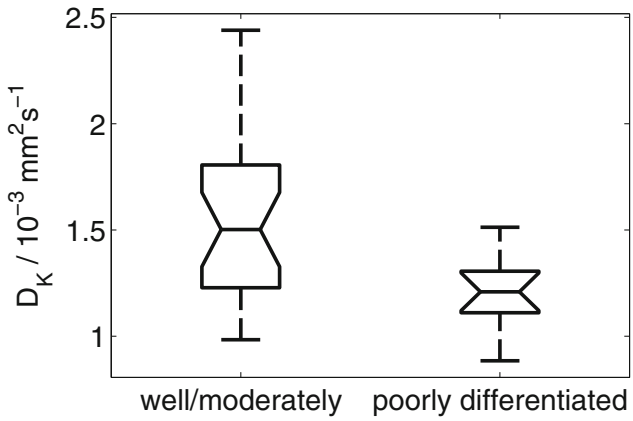

b

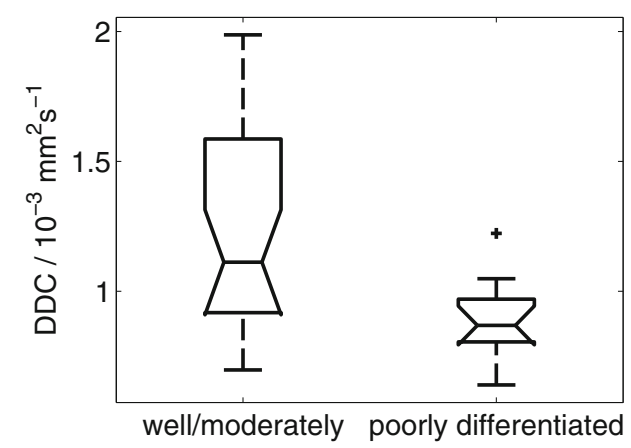

d

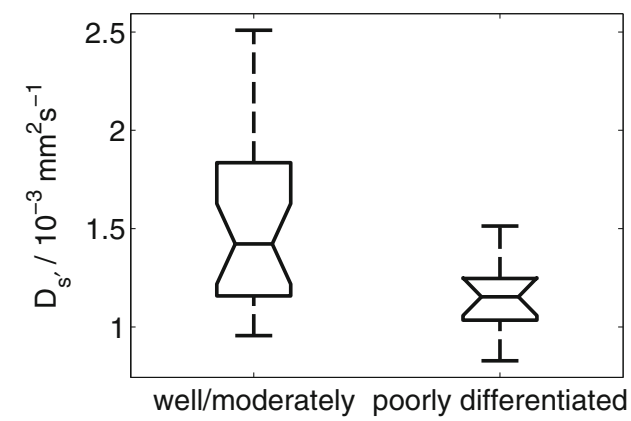

e

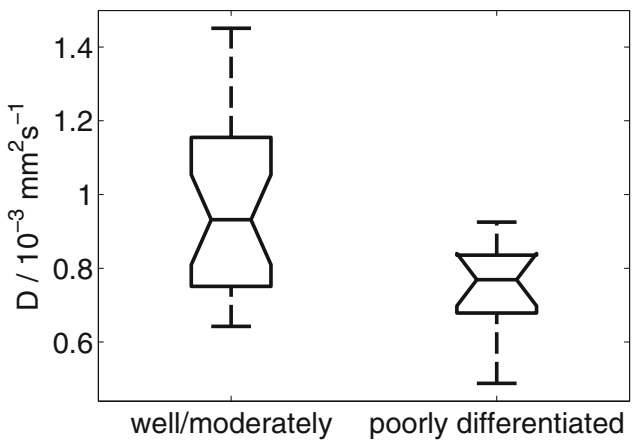

previous studies [21, 22]. Diffusion coefficients from nonmono-exponential models (DDC, $\mathrm{D}_{\mathrm{K}}, \mathrm{D}_{\mathrm{s}^{\prime}}$, and $\left.\mathrm{D}\right)$ showed similar results to $\mathrm{ADC}$, with lower estimates in poorly differentiated tumours compared with well/moderately differentiated tumours. These results, together with the strong correlation between ADC and these other diffusion coefficients, indicate that these parameters provide similar information to $\mathrm{ADC}$. The parameter $\mathrm{D}_{\mathrm{s}}$ from the statistical model was an exception to this behaviour, exhibiting only weak correlation with ADC and no significant difference between tumour grades. Median estimates of $D_{s}$ included many values close to zero, as described in other studies [29]. After reformulating $D_{s}$ to $D_{s}$, however, a significant difference between tumour grades was observed, and the median estimates matched ADC estimates more closely. It is notable that ADC and other diffusion coefficients differentiated grades of tumours where the major histological feature is increasing cell density and mitoses with increasing grade but that other parameters best differentiated squamous cell carcinoma and adenocarcinoma where structural features are more profound and rely on cell organisation and glandular architecture rather than cell density alone. Unfortunately, only two poorly differentiated adenocarcinomas were present in this cohort of patients due to the lower numbers of adenocarcinomas compared with squamous cell carcinomas in cancers of the uterine cervix [30].

Considering correlation of parameters within models, the absence of correlation between $\alpha$ and DDC from the stretched exponential model, $\sigma$ and $\mathrm{D}_{\mathrm{s}}$ from the statistical model, and 


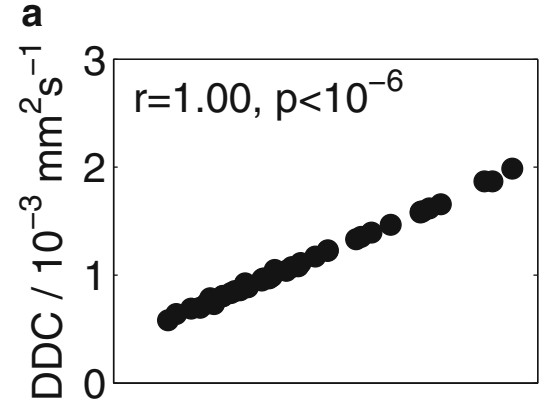

b

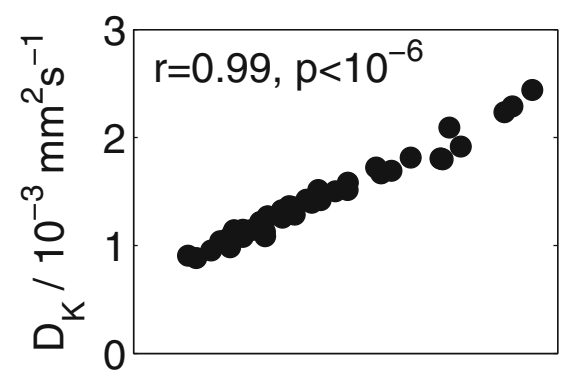

C
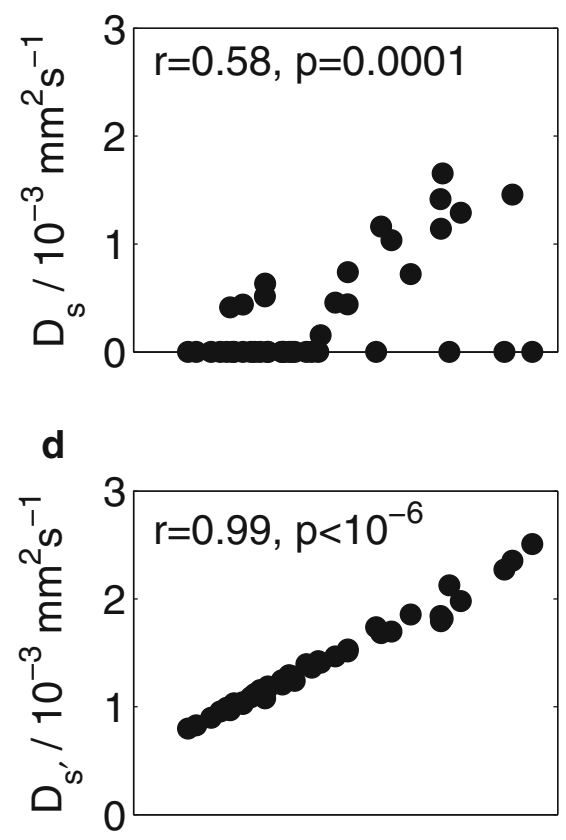

e

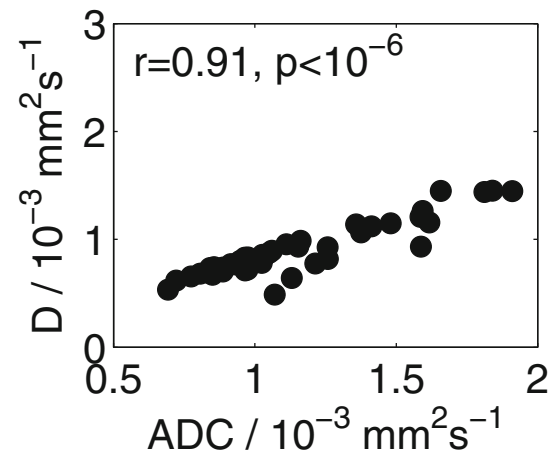

4 Fig. 4 Correlation between ADC and a DDC from the stretched exponential model, $\mathbf{b} \mathrm{D}_{\mathrm{K}}$ from the kurtosis model, $\mathbf{c} \mathrm{D}_{\mathrm{s}}$ from the statistical model, $\mathbf{d} \mathrm{D}_{\mathrm{s}^{\prime}}$ from the statistical model, and $\mathbf{e} \mathrm{D}$ from the biexponential model

either $f$ and $\mathrm{D}$ or $\mathrm{D}^{*}$ and $\mathrm{D}$ from the bi-exponential model indicates that these parameters provide complementary information and may be sensitive to different aspects of the tumour micro-structure. The strong correlation observed between $\sigma^{\prime}$ and $\mathrm{D}_{\mathrm{s}^{\prime}}$ from the reformulated statistical model, however, indicates that the additional parameter $\sigma^{\prime}$ may not provide additional information. The correlation between $\mathrm{K}$ and $\mathrm{D}_{\mathrm{K}}$ from the kurtosis model indicates that these parameters also provide largely the same information, but in this case the additional parameter does provide some additional information as $\mathrm{K}$ and $\mathrm{D}_{\mathrm{K}}$ were sensitive to different properties (type and grade, respectively).

Repeatability of fitted parameters is an important consideration in model selection. It was not possible to carry out repeated examinations on the patients in this study owing to the invasive nature of the MRI examination using an endovaginal receiver coil and it was, therefore, not possible to assess the test-retest repeatability of the parameter estimates. However, the repeatability of fitted parameters of the bi-exponential and stretched exponential models, which were preferred by largest numbers of tumours in this study, have been investigated in several previous studies that use similar DW-MRI protocols to the one used here $[9,11,13$, 31]. The parameters of the stretched exponential model have been shown to have very good repeatability (coefficient of variation $(\mathrm{CV}) \sim 4$ to $7 \%$ ), comparable to the repeatability of ADC estimates $[9,11,13]$, while the repeatability of parameters from the bi-exponential model, particularly $f$ and $\mathrm{D}^{*}$ are significantly worse $(\mathrm{CV}>20 \%)[9,11$, $13,31]$. Whilst the ANOVA $p$-values indicate that $f$ and $\mathrm{D}^{*}$ show greater significance than $\alpha$ and $\mathrm{K}$ when making group comparisons between tumour type, the poorer repeatability of $f$ and $\mathrm{D}^{*}$ suggest that $\alpha$ and $\mathrm{K}$ may be more appropriate for differentiating tumour types in individuals.

An apparent limitation of this study is the use of a maximum b-value of $800 \mathrm{~s} \mathrm{~mm}^{-2}$ when applying models that were originally developed over much wider ranges of bvalues $[4,5]$. A maximum b-value of $800 \mathrm{~s} \mathrm{~mm}^{-2}$ is, however, typical of DW-MRI protocols in body applications where higher b-values increase the minimum TE attainable, thus lowering signal-to-noise ratio (SNR), and increase image distortion due to eddy current effects. The lack of bvalues over $1000 \mathrm{~s} \mathrm{~mm}^{-2}$ would be a severe limitation in investigations of intra- and extra-cellular compartments in the brain, where some of the models investigated here were originally developed $[4,5]$. In this study, however, the modelling process was a phenomenological investigation in order to assess the best description of the data and the 
Table 3 Correlation between fitted parameters within each nonmono-exponential model

\begin{tabular}{llcl}
\hline Model & Parameters & Pearson's correlation coefficient $(\mathrm{r})$ & $p$-value \\
\hline Stretched exponential & $\mathrm{DDC}, \alpha$ & 0.14 & 0.4 \\
Kurtosis & $\mathrm{D}_{\mathrm{K}}, \mathrm{K}$ & -0.72 & $<10^{-6} *$ \\
Statistical & $\mathrm{D}_{\mathrm{s}}$ (mode), $\sigma$ (scale parameter) & -0.13 & 0.4 \\
& $\mathrm{D}_{\mathrm{s}^{\prime}}$ (mean), $\sigma^{\prime}$ (s.d.) & 0.91 & $<10^{-6} *$ \\
Bi-exponential & $\mathrm{D}, f$ & 0.28 & 0.08 \\
& $\mathrm{D}, \mathrm{D} *$ & 0.18 & 0.2 \\
& $\mathrm{D}^{*}, f$ & -0.37 & $0.02 *$ \\
& $\mathrm{D}, f \mathrm{D}^{*}$ & 0.64 & $5 \times 10^{-6} *$ \\
\hline
\end{tabular}

Table shows Pearson's linear correlation coefficient (r) and $p$-value for each pair of parameters. $* p<0.05$ original theoretical formulations were not implied. Also, although grading of tumours was carried out on cone biopsy in 30/42 cases, this represented a significant volume of tumour and mitigates against variations in tumour grade on needle biopsy.

In conclusion, non-mono-exponential models were preferred to the mono-exponential model in DW-MRI data from cervical tumours. ADC and the diffusion coefficients from other models were significantly different between grades of tumours, but showed no differences between tumour types, while $\alpha$ from the stretched exponential model, $\mathrm{K}$ from the kurtosis model, and $f$ and $\mathrm{D}^{*}$ from the bi-exponential model were significantly different between squamous cell carcinomas and adenocarcinomas. These results show that parameters from non-mono-exponential models are related to different aspects of tumour microstructure. Parameters from nonmono-exponential models may, therefore, have utility in probing features of tumour phenotype, which may be indicative of poor prognosis or progressive disease. Of the models tested, the stretched exponential model exhibited uncorrelated parameters, which were related to histological features and is, therefore, likely to be most relevant for clinical use.

Acknowledgments The scientific guarantor of this publication is Professor Nandita deSouza, Professor of Translational Imaging, MRI Unit, Institute of Cancer Research and Royal Marsden Hospital, Downs Road, Sutton, Surrey. SM2 5PT. UK. The authors of this manuscript declare no relationships with any companies, whose products or services may be related to the subject matter of the article. This study has received funding by CRUK and EPSRC support to the Cancer Imaging Centre at ICR and RMH in association with MRC and Department of Health C1060/A10334, C1060/A16464 and NHS funding to the NIHR Biomedical Research Centre and the Clinical Research Facility in Imaging. No complex statistical methods were necessary for this paper. Institutional Review Board approval was obtained. Written informed consent was obtained from all subjects (patients) in this study. Methodology: prospective, observational, performed at one institution.

Open Access This article is distributed under the terms of the Creative Commons Attribution 4.0 International License (http:// creativecommons.org/licenses/by/4.0/), which permits unrestricted use, distribution, and reproduction in any medium, provided you give appropriate credit to the original author(s) and the source, provide a link to the Creative Commons license, and indicate if changes were made.

\section{References}

1. Le Bihan D, Breton E, Lallemand D, Aubin M-L, Vignaud J, LavalJeantet M (1988) Separation of diffusion and perfusion in intravoxel incoherent motion MR imaging. Radiology 168:497-505

2. Bennett KM, Schmainda KM, Bennett (Tong) R, Rowe DB, Lu H, Hyde JS (2003) Characterization of continuously distributed cortical water diffusion rates with a stretched-exponential model. Magn Reson Med 50:727-734

3. Yablonskiy DA, Bretthorst GL, Ackerman JJH (2003) Statistical model for diffusion attenuated MR signal. Magn Reson Med 50: 664-669

4. Jensen JH, Helpern JA, Ramani A, Lu H, Kaczynski K (2005) Diffusional kurtosis imaging: the quantification of non-gaussian water diffusion by means of magnetic resonance imaging. Magn Reson Med 53:1432-1440

5. Kiselev VG, Il'yasov KA (2007) Is the "biexponential diffusion" biexponential? Magn Reson Med 57:464-469

6. Riches SF, Hawtin K, Charles-Edwards EM, deSouza NM (2008) Diffusion-weighted imaging of the prostate and rectal wall: comparison of biexponential and monoexponential modelled diffusion and associated perfusion coefficients. NMR Biomed 22:318-325

7. Jansen JFA, Stambuk HE, Koutcher JA, Shukla-Dave A (2010) Non-gaussian analysis of diffusion-weighted MR imaging in head and neck squamous cell carcinoma: a feasibility study. AJNR Am J Neuroradiol 31:741-748

8. Rosenkrantz AB, Sigmund EE, Johnson G et al (2012) Prostate cancer: feasibility and preliminary experience of a diffusional kurtosis model for detection and assessment of aggressiveness of peripheral zone cancer. Radiology 264:126-135

9. Mazaheri Y, Afaq A, Rowe DB, Lu Y, Shulka-Dave A, Grover J (2012) Diffusion-weighted magnetic resonance imaging of the prostate: improved robustness with stretched exponential modeling. J Comput Assist Tomogr 36:695-703

10. Bourne RM, Panagiotaki E, Bongers A, Sved P, Watson G, Alexander DC (2014) Information theoretic ranking of four models of diffusion attenuation in fresh and fixed prostate tissue ex vivo. Magn Reson Med 72:1418-1426

11. Winfield JM, deSouza NM, Priest AN et al (2015) Modelling DWMRI data from primary and metastatic ovarian tumours. Eur Radiol 25:2033-2040

12. Hauser T, Essig M, Jensen A et al (2013) Characterization and therapy monitoring of head and neck carcinomas using diffusionimaging-based intravoxel incoherent motion parameters - preliminary results. Neuroradiology 55:527-536

13. Orton MR, Messiou C, Collins D et al (2015) Diffusion-weighted MR imaging of metastatic abdominal and pelvic tumours is sensitive to early changes induced by a VEGF inhibitor using alternative diffusion attenuation models. Eur Radiol. doi:10.1007/s00330-015-3933-7 
14. Norris DG (2001) The effects of microscopic tissue parameters on the diffusion weighted magnetic resonance imaging experiment. NMR Biomed 14:77-93

15. Yablonskiy DA, Sukstanskii AL (2010) Theoretical models of the diffusion weighted MR signal. NMR Biomed 23:661-681

16. Koh D-M, Collins DJ, Orton MR (2011) Intravoxel incoherent motion in body diffusion-weighted MRI: reality and challenges. AJR Am J Roentgenol 196:1351-1361

17. Rosenkrantz AB, Padhani AR, Chenevert TL et al (2015) Body diffusion kurtosis imaging: basic principles, applications, and considerations for clinical practice. J Magn Reson Imaging 42:1190-1202

18. Charles-Edwards EM, Messiou C, Morgan VA et al (2008) Diffusion-weighted imaging in cervical cancer with an endovaginal technique: potential value for improving tumor detection in stage Ia and $\mathrm{Ib} 1$ disease. Radiology 249:541-550

19. Downey K, Jafar M, Attygalle AD et al (2013) Influencing surgical management in patients with carcinoma of the cervix using a T2and ZOOM-diffusion-weighted endovaginal MRI technique. Br J Cancer 109:615-622

20. Downey K, Attygalle AD, Morgan VA et al (2015) Comparison of optimised endovaginal vs external array coil T2-weighted and diffusion-weighted imaging techniques for detecting suspected early stage (IA/IB1) uterine cervical cancer. Eur Radiol. doi:10.1007/s00330-015-3899-5

21. Payne GS, Schmidt M, Morgan VA et al (2010) Evaluation of magnetic resonance diffusion and spectroscopy measurements as predictive biomarkers in stage 1 cervical cancer. Gynecol Oncol 116:246-252
22. Kuang F, Ren J, Zhong Q, Liyuan F, Huan Y, Chen Z (2013) The value of apparent diffusion coefficient in the assessment of cervical cancer. Eur Radiol 23:1050-1058

23. Lee EPL, Yu X, Chu MYC et al (2014) Perfusion and diffusion characteristics of cervical cancer based on intravoxel incoherent motion MR imaging - a pilot study. Eur Radiol 24:1506-1513

24. deSouza NM, Scoones D, Krausz T, Gilderdale DJ, Soutter WP (1996) High resolution MR imaging of stage 1 cervical neoplasia with a dedicated transvaginal coil: MR features and correlation of imaging and pathologic findings. AJR Am J Roentgenol 166:553-559

25. Blackledge M, Leach M, Collins D, Koh D-M (2011) Computed diffusion-weighted MR imaging may improve tumour detection. Radiology 261:573-581

26. Barr DR, Sherrill ET (1999) Mean and variance of truncated normal distributions. Am Stat 53:357-361

27. Schwarz G (1978) Estimating the dimension of a model. Ann Stat 6:461-464

28. Panek R, Borri M, Orton M et al (2015) Evaluation of diffusion models in breast cancer. Med Phys 42:4833-4839

29. Yuan J, Yeung DKW, Mok GSP et al (2014) Non-Gaussian analysis of diffusion weighted imaging in head and neck at 3T: a pilot study in patients with nasopharyngeal carcinoma. PLoS ONE 9, e87024

30. Waggoner SE (2003) Cervical cancer. Lancet 361:2217-2225

31. Andreou A, Koh DM, Collins DJ et al (2013) Measurement reproducibility of perfusion fraction and pseudodiffusion coefficient derived by intravoxel incoherent motion diffusion-weighted MR imaging in normal liver and metastases. Eur Radiol 23:428-434 